University of Windsor

Scholarship at UWindsor

1991

\title{
Variational calculation for the ground state of lithium and the QED corrections for Li-like ions
}

D. K. McKenzie

Gordon W. F. Drake

University of Windsor

Follow this and additional works at: https://scholar.uwindsor.ca/physicspub

Part of the Physics Commons

\section{Recommended Citation}

McKenzie, D. K. and Drake, Gordon W. F.. (1991). Variational calculation for the ground state of lithium and the QED corrections for Li-like ions. Physical Review A, 44 (11), R6973-R6976.

https://scholar.uwindsor.ca/physicspub/79

This Article is brought to you for free and open access by the Department of Physics at Scholarship at UWindsor. It has been accepted for inclusion in Physics Publications by an authorized administrator of Scholarship at UWindsor. For more information, please contact scholarship@uwindsor.ca. 


\title{
PHYSICAL REVIEW A
}

\section{ATOMIC, MOLECULAR, AND OPTICAL PHYSICS}

\section{RAPID COMMUNICATIONS}

The Rapid Communications section is intended for the accelerated publication of important new results. Since manuscripts submitted to this section are given priority treatment both in the editorial office and in production, authors should explain in their submittal letter why the work justifies this special handling. A Rapid Communication should be no longer than 31/2 printed pages and must be accompanied by an abstract. Page proofs are sent to authors.

\section{Variational calculation for the ground state of lithium and QED corrections for Li-like ions}

\author{
D. K. McKenzie and G. W. F. Drake \\ Department of Physics, University of Windsor, Windsor, Ontario, Canada N9B 3P4
}

(Received 29 August 1991)

\begin{abstract}
High-precision variational calculations using multiple basis sets in Hylleraas coordinates are presented for the $1 s^{2} 2 s^{2} S$ state of lithium. The variational bound of -7.478060326(10) a.u. for the nonrelativistic energy is in good agreement with our revised experimental value of $-7.47806034(20)$ a.u., thereby resolving a long-standing discrepancy. Two-electron calculations of the QED corrections are extended to three-electron systems and compared with other results. The comparison for Li-like ions up to $\mathrm{U}^{89+}$ suggests a simple interpretation for the "screening of the Lamb shift" recently calculated by Cheng, Johnson, and Sapirstein [Phys. Rev. Lett. 66, 2960 (1991)].
\end{abstract}

PACS number(s): 31.20.Di, 31.30.Jv

Lithium is one of the fundamental few-body problems of atomic physics. However, theoretical calculations for the nonrelativistic energy of the ground state appear to lie substantially above the experimental value (corrected for relativistic and QED effects), despite the efforts of a long sequence of authors to obtain improved variational upper bounds [1-5]. The discrepancy was most recently discussed by Chung [4], but no resolution to the problem was suggested.

The purpose of this paper is twofold. The first is to present a variational method employing multiple basis sets in Hylleraas coordinates. Unlike previous variational calculations with large basis sets, a systematic procedure for enlarging the basis set is imposed so that the convergence of the results and their extrapolation to larger basis sets have a well-defined meaning. The method yields a substantial improvement in the variational bound to the nonrelativistic energy. The second is to show that a revised analysis of the relativistic and QED corrections removes the discrepancy of about $10 \mu \mathrm{a}$.u. between theory and experiment. The accuracy of the QED correction is checked by comparison with other experimental and theoretical results up to $\mathrm{U}^{89+}$, and a simple interpretation of the "screening of the Lamb shift" is suggested.

Past high-precision calculations for lithium have been based on variational calculations in Hylleraas coordinates
[2,3], the superposition of correlated configurations (SCC) method [1], or multiconfiguration-interaction wave functions $[4,5]$. The present method developed as a natural extension of the double-basis-set calculations for helium [6], which have been found to yield a dramatic improvement in accuracy for a given number of terms. It can be thought of as a hybrid between a pure Hylleraas calculation and the SCC method.

In the present calculation, the wave function for the $1 s^{2} 2 s^{2} S$ state is written in the form

$$
\psi\left(\mathbf{r}_{1}, \mathbf{r}_{2}, \mathbf{r}_{3}\right)=\mathcal{A} \sum_{v=1}^{6} \sum_{\mu_{v}} C_{v, \mu_{v}} \phi_{v, \mu_{v}} \chi_{1},
$$

where the spatial part of the wave function, $\phi_{v, \mu_{v}}$, is of the form

$$
\begin{aligned}
& \phi_{v, \mu_{v}}\left(r_{1}, r_{2}, r_{3}, r_{23}, r_{31}, r_{12}\right) \\
& \quad=r_{1}^{m_{1}} r_{2}^{m_{2}} r_{3}^{m_{3}} r_{23}^{n_{23}} r_{31}^{n_{31}} r_{12}^{n_{12}} \exp \left(-\alpha_{v} r_{1}-\beta_{v} r_{2}-\gamma_{v} r_{3}\right),
\end{aligned}
$$

where $\mu_{v}$ denotes a sextuple of integer powers $\left(m_{1}, m_{2}\right.$, $\left.m_{3}, n_{23}, n_{31}, n_{32}\right)$ and the spin function $\chi_{1}$ is

$$
\chi_{1}=\alpha(1) \beta(2) \alpha(3)-\beta(1) \alpha(2) \alpha(3) .
$$

As shown by Larsson [7], the second linearly independent 
TABLE I. Nonrelativistic eigenvalues for the $1 s^{2} 2 s^{2} S$ state of $\mathrm{Li}$.

\begin{tabular}{ccll}
\hline \hline$K$ & No. of terms & \multicolumn{1}{c}{$E(K)$ (a.u.) } & $E(K)-E(K-1)$ \\
\hline 1 & 18 & -7.476946306762 & \\
2 & 48 & -7.4778667203778 & -0.000920413626 \\
3 & 108 & -7.478041912521 & -0.000175192133 \\
4 & 216 & -7.47805884395 & -0.00001693143 \\
5 & 396 & -7.4780601265 & -0.0000012825 \\
6 & 690 & -7.4780602661 & -0.0000001396 \\
7 & 1134 & -7.478060312 & -0.000000046 \\
& Extrapolation & $-7.478060326(10)$ & $-0.000000014(10)$ \\
\hline \hline
\end{tabular}

spin function need not be explicitly included, provided that none of the electrons is constrained to be equivalent. $\mathcal{A}$ represents the three-particle antisymmetrizer, and $v$ labels different sets of exponential parameters $\alpha_{v}, \beta_{v}$, and $\gamma_{v}$ used with the combinations of powers labeled by $\mu_{v}$.

In order to keep the basis set reasonably compact, the combinations of powers associated with each of the six values of $v$ were determined by the inequalities

$$
m_{i} \leq m_{j} \leq m_{k}, n_{i j} \leq n_{i k} \leq n_{j k}
$$

for the six permutations of the labels $(i, j, k)=(1,2,3)$. Thus, for example, the term $(0,0,0,0,0,0,0)$ was included six times with different values for $\boldsymbol{\alpha}_{v}, \boldsymbol{\beta}_{v}$, and $\gamma_{v}$ in each case. The latter were determined by calculating analytically [6] the eighteen derivatives $\partial E / \partial \alpha_{v}, \partial E / \partial \beta_{v}$, and $\partial E / \partial \gamma_{v}, v=1, \ldots, 6$, and locating the zeros on the multidimensional energy surface. Finally, the basis set was systematically enlarged by including all combinations of powers consistent with (4) such that

$$
m_{1}+m_{2}+m_{3}+n_{23}+n_{31}+n_{12} \leq K,
$$

and then progressively increasing the integer $K$. As with the two-electron case [6], the optimization of the nonlinear parameters leads to a natural partition of the basis set into parts representing the asymptotic, intermediate range, and inner correlation parts of the wave function. It is important to continue reoptimizing as $K$ increases in order to avoid problems of near linear dependence in the basis set. The results presented here are for a series of calculations for $K$ up to seven, producing a basis set of 1134 terms.

The results of the calculations are shown in Table I. The best variational upper bound to the nonrelativistic energy is -7.478060312 a.u., with an extrapolated energy of $-7.478060326(10)$ a.u. The extrapolated value was obtained from ratios of successive differences. These results represent an improvement in accuracy of two significant figures over the previous calculations listed in Table II. As an example of the improvement gained with our multiple basis sets, the 216-term result in Table $I$ is comparable in accuracy to the 602-term result of King [2]. The best previous bound of King and Bergsbaken [3] was obtained by individually optimizing the exponents for each basis-set member as it was added. The configuration-interaction result of Jitrik and Bunge [5] was obtained by a large $(-149.373 \mu$ a.u. $)$ extrapolation of their $K$-shell energies. The lower value they obtain is probably an artifact of this extrapolation.

Our present result still lies $12.7 \mu$ a.u. below the estimate -7.478073 a.u. obtained by Bunge [8] from the experimental ionization energy [9] and used by most other authors since then. In order to resolve this discrepancy, we present in Table III a revised estimate. The contributions from relativistic, mass polarization, and QED effects to the ionization energy are subtracted, and then the accurately known $1 s^{2} S$ energy of $\mathrm{Li}^{+}[10]$ is added to obtain $-7.47806034(20)$ a.u., in agreement with our calculation. The principal reason for Bunge's lower value is that he counted twice the $K$-shell mass polarization correction of $22.60 \mu$ a.u., which was already included in the $K$-shell correction used by him. This is partially offset by our larger value for the QED correction, as further discussed below.

Our calculation of the one- and two-electron QED corrections $\Delta E_{L, 1}, \Delta E_{L, \sigma}$, and $\Delta E_{L, 2}$ in Table III is an extension of a method developed previously for two-electron ions $[11,12]$. It differs substantially from previous estimates for the lithium isoelectronic sequence, except for recent work by Indelicato and Desclaux [13]. $\Delta E_{L, 1}$ is given (in a.u.) by

$$
\begin{aligned}
\Delta E_{L, 1}=\alpha^{3} Z & {\left[\left(\frac{x F\left(1 s_{1 / 2}\right)+F\left(n l_{j}\right) / n^{3}}{x+\delta_{l, 0} / n^{3}}\right]\right.} \\
& \left.\times\left\langle\sum_{i} \delta\left(\mathbf{r}_{i}\right)\right\rangle_{1 s^{x} n l}-F\left(1 s_{1 / 2}\right)\left\langle\sum_{i} \delta\left(\mathbf{r}_{i}\right)\right\rangle_{1 s^{x}}\right],
\end{aligned}
$$

where $x=1$ or 2 is the number of $1 s$ electrons and $F\left(n l_{j}\right)$ is the one-electron QED function tabulated by Johnson and Soff [14]. The above follows rigorously from the assumption that the two- or three-electron QED shift is the same as the one-electron QED shift corrected for the elec-

TABLE II. Comparison with other recent calculations for the nonrelativistic ground-state energy of Li.

\begin{tabular}{lcl}
\hline \multicolumn{1}{c}{ Author } & Method & \multicolumn{1}{c}{$E$ (a.u.) } \\
\hline Kleindienst and Beutner [1] & 310-term SCC & -7.47805824 \\
King [2] & 602-term Hylleraas & -7.478059 \\
King and Bergsbaken [3] & 296-term Hylleraas & -7.478059528 \\
Chung [4] & Extrapolated CI & -7.4780597 \\
Present work & 1134-term Hylleraas & $-7.478060326(10)$ \\
Jitrik and Bunge [5] & Extrapolated CI & $-7.47806241(72)$ \\
\hline \hline
\end{tabular}


TABLE III. Nonrelativistic energy for the ground state of lithium. Corrections are relative to $\mathrm{Li}^{+}\left(1 s^{2} S\right)$.

\begin{tabular}{lc}
\hline \hline \multicolumn{1}{c}{ Contribution } & Value (a.u.) \\
\hline$E\left(1 s^{2} 2 s^{2} S\right)-E\left(1 s^{2}{ }^{1} S\right)$ & $-0.19815753(2)^{\mathrm{a}}$ \\
$-\Delta E_{\text {Breit }}$ & $0.00001258(10)^{\mathrm{b}}$ \\
$-\Delta E_{\text {Mass pol. }}$ & $-0.00000100^{\mathrm{b}}$ \\
$-\Delta E_{L, 1}$ & $-0.00000139^{\mathrm{c}}$ \\
$-\Delta E_{L, \sigma}$ & $0.00000021(5)^{\mathrm{c}}$ \\
$-\Delta E_{L, 2}$ & $0.00000020(10)^{\mathrm{c}}$ \\
$E_{\mathrm{NR}}\left(1 s^{2}{ }^{\mathrm{I}} S\right)$ & $-7.2799134126693^{\mathrm{d}}$ \\
Total & $-7.47806034(20)$ \\
\hline \hline
\end{tabular}

aFrom the experimental ionization energy [9], using $R_{M}$ $=109728.7340 \mathrm{~cm}^{-1}$.

${ }^{\text {b}}$ Chung, in Ref. [4].

'Present work.

${ }^{\mathrm{d}}$ Freund, Huxtable, and Morgan [10] and G. W. F. Drake (unpublished).

tron density at the nucleus. This is exactly correct for the vacuum-polarization term, at least to lowest order in $\alpha$, because this term is the expectation value of a short-range potential at the nucleus. The above also gives correctly the leading term in a $1 / Z$ expansion of the Bethe logarithm [12]. The next to leading term, $\Delta E_{L, \sigma}$, is [12]

$$
\Delta E_{L, \sigma}=-\frac{4}{3} \alpha^{3} Z\left[\ln \left(\frac{Z-\sigma\left(1 s^{x} n l\right)}{Z}\right)^{2}\left\langle\sum_{i} \delta\left(\mathbf{r}_{i}\right)\right\rangle_{l s^{x} n l}-\ln \left(\frac{Z-\sigma\left(1 s^{x}\right)}{Z}\right)^{2}\left\langle\sum_{i} \delta\left(\mathbf{r}_{i}\right)\right\rangle_{1 s^{x}}\right]
$$

with $\sigma\left(1 s^{1}\right)=0$. The $\sigma^{\prime}$ s are screening constants related to the $1 / Z$ expansion coefficients $A_{i}$ and $B_{i}$ for the numerator and denominator of the Bethe logarithm [12] by

$$
\sigma=-\left(A_{1} B_{0}-A_{0} B_{1}\right) /\left(2 B_{0}\right)^{2}
$$

for a given $1 s^{x} n l$ configuration. It follows from the two-particle coefficients of fractional parentage [15] that

$$
\begin{aligned}
& A_{0}\left(1 s^{2} n l^{2} L\right)=\frac{1}{2}\left[A_{0}\left(1 s^{2}{ }^{1} S\right)+\frac{1}{2} A_{0}\left(1 s n l{ }^{1} L\right)+\frac{3}{2} A_{0}\left(1 s n l^{3} L\right)\right], \\
& A_{1}\left(1 s^{2} n l^{2} L\right)=\left[A_{1}\left(1 s^{2} S\right)+\frac{1}{2} A_{1}\left(1 s n l^{1} L\right)+\frac{3}{2} A_{1}\left(1 s n l^{3} L\right)\right],
\end{aligned}
$$

and similarly for $B_{0}$ and $B_{1}$. Numerical values for the two-electron states [12] then give immediately $\sigma\left(1 s^{2} 2 s^{2} S\right)=-0.00842(1)$, and $\sigma\left(1 s^{2} 2 p^{2} P\right)=0.00165(1)$. These are direct perturbation calculations, not empirical fits to data. For the $\delta$-function matrix elements, the above analysis gives the $1 / Z$ expansions

$$
\begin{aligned}
& \pi\left\langle\sum_{i} \delta\left(\mathbf{r}_{i}\right)\right\rangle_{1 s^{2}}=2 Z^{3}\left(1-0.6676396 Z^{-1}+0.177 Z^{-2}\right), \\
& \pi\left\langle\sum_{i} \delta\left(\mathbf{r}_{i}\right)\right\rangle_{1 s^{2} 2 s}=\frac{17}{8} Z^{3}\left(1-0.8438476 Z^{-1}+0.345 Z^{-2}\right), \\
& \pi\left\langle\sum_{i} \delta\left(\mathbf{r}_{i}\right)\right\rangle_{1 s^{2} 2 p}=2 Z^{3}\left(1-0.7266416 Z^{-1}+0.297 Z^{-2}\right) .
\end{aligned}
$$

The leading two terms are the numerical values for $B_{0} / 2$ and $B_{1} / 2$. The last term in Eqs. (11) and (12) were obtained by fitting to high-precision calculations [2,16] used for $Z \leq 10$. Equations (11)-(13) become essentially exact for $Z \geq 7$, and are useful for extrapolating to higher $Z$.

The remaining explicit two-electron QED correction is

$$
\Delta \dot{E}_{L, 2}=\alpha^{3}\left(\frac{14}{3} \ln \alpha+\frac{164}{15}\right)\left[\left\langle\delta\left(\mathbf{r}_{i, j}\right)\right\rangle_{1 s^{2} n l}-\left\langle\delta\left(\mathbf{r}_{1,2}\right)\right\rangle_{1 s^{2}}\right]-\frac{14}{3} \alpha^{3}\left[Q\left(1 s^{2} n l\right)-Q\left(1 s^{2}\right)\right],
$$

where $Q=(1 / 4 \pi) \lim _{a \rightarrow 0}\left\langle r_{i, j}{ }^{3}(a)+4 \pi(\gamma+\ln a) \delta\left(\mathbf{r}_{i, j}\right)\right\rangle, \gamma$ is Euler's constant, $a$ is the radius of a sphere about $r_{i, j}=0$ excluded from the integration, and a summation over $i>j$ from 1 to 3 is assumed. Combining the two-electron results from Ref. [11] results in

$$
\begin{aligned}
& Q\left(1 s^{2} 2 s\right)=-\left\langle\delta\left(\mathbf{r}_{i, j}\right)\right\rangle\left(\ln Z+\frac{1}{6} \ln 3+\frac{7}{12} \ln 2-1\right)+0.01446 Z^{3}+O\left(Z^{2}\right), \\
& Q\left(1 s^{2} 2 p\right)=-\left\langle\delta\left(\mathbf{r}_{i, j}\right)\right\rangle\left(\ln Z+\frac{1}{6} \ln 3+\frac{7}{12} \ln 2-1\right)+0.01762 Z^{3}+O\left(Z^{2}\right), \\
& Q\left(1 s^{2}\right)=-\left\langle\delta\left(\mathbf{r}_{1,2}\right)\right\rangle(\ln Z+\ln 2-1)+0.01326 Z^{3}+O\left(Z^{2}\right) .
\end{aligned}
$$


Accurate values of $\left\langle\delta\left(\mathbf{r}_{i, j}\right)\right\rangle$ were obtained from $\left\langle\delta\left(\mathbf{r}_{i, j}\right)\right\rangle$ $=H_{3} / \pi \alpha^{2}$, where $H_{3}$ is the spin-spin term tabulated by Chung [4]. Although this term increases only in proportion to $Z^{3}$, it is a dominant source of uncertainty for neutral $\mathrm{Li}$, and accurate values of $Q$ will be required in order to make further progress in the comparison with experiment.

The total QED correction shown in Table III is about three times larger than that used by Chung [4] because his effective nuclear charge $Z_{\text {eff }} \simeq Z-1.6$, obtained from the ionization energy, overestimates the screening of the electron density at the nucleus. His smaller value would produce a serious disagreement between theory and experiment for the total nonrelativistic energy. For the higher- $Z$ ions, our values are about twice as large as Chung's. They are listed in Table IV. This more than compensates for the discrepancies he found, and produces a somewhat larger discrepancy in the reverse direction. For example, at $Z=10$, replacing Chung's QED correction by the one given in Table IV yields a predicted ionization potential of $1928436 \mathrm{~cm}^{-1}$, in comparison with the experimental value $1928462 \mathrm{~cm}^{-1}$.

The accuracy of our calculated QED term can be tested in several ways. First, the above terms for the case $x=1$ predict Lamb shifts for the $1 s 2 s 1 S$ and ${ }^{3} S$ states of heliumlike ions to an accuracy of $\pm 5 \%$ or better over a wide range of nuclear charge $[11,17,18]$. Second, many-body perturbation theory (MBPT) calculations of the selfenergy and vacuum-polarization terms for three-electron $\mathrm{U}^{89+}$ have recently been performed [19]. Applying Eqs. (6) and (7) to this case, together with (11)-(13), for $\delta$ function matrix elements results in $\Delta E_{L, 1+\sigma}\left(1 s^{2} 2 s^{2} S_{1 / 2}\right)$ $=1.730$ a.u., and $\Delta E_{L, 1+\sigma}\left(1 s^{2} 2 p^{2} P_{1 / 2}\right)=0.224$ a.u. for the sum of the self-energy and vacuum-polarization parts, including their finite-nuclear-size corrections as tabulated in Ref. [14]. This is in excellent agreement with the MBPT values of $1.724(2)$ and $0.220(2)$ a.u., respectively, for the same terms. Including the other higher-order terms in Ref. [19] results in a total ${ }^{2} P_{1 / 2}{ }^{2} S_{1 / 2}$ Lamb shift of $-41.62(15) \mathrm{eV}$, in agreement with the experimental value $-41.65(10) \mathrm{eV}$ [20]. The above shows that the "screening of the Lamb shift" calculated by MBPT [19] can be largely understood as a renormalization of the nonrelativistic electron density at the nucleus relative to the one-electron value, together with a small contribution from the $\Delta E_{L, \sigma}$ term. Finally, Table IV compares the calculated $2{ }^{2} P_{1 / 2}-2{ }^{2} S_{1 / 2}$ QED correction with the "experimental" values obtained by Johnson, Blundell, and Sapirstein [21]. The calculated values are systematically larger, but the differences are only about $3.5 \%$. For $Z>15$, our values are systematically larger than those of Indelicato and Desclaux [13] by about $1.5 \%$. The results depend sensitively on $\left\langle\delta\left(\mathbf{r}_{i}\right)\right\rangle$ and estimates from Eq. (13) for the $2^{2} P_{1 / 2}$ state are not reliable for $Z<7$.

In summary, the results of this paper show that the ionization energy of lithium is now understood at the 0.2 $\mu$ a.u. level of accuracy. A much improved variational bound for the nonrelativistic energy has been obtained, and a long-standing discrepancy with experiment has been resolved. However, discrepancies remain for the ionization potentials of the $\mathrm{Li}$-like ions which require further study.

Note added. After completion of this work, we learned of an SCC calculation by J. Pipin and D. M. Bishop (unpublished). Their ground-state energy for $\mathrm{Li}$ is -7.4780601 a.u.

We wish to thank Kwong Chung for making his results available in advance of publication, and Jonathan Sapirstein for helpful comments. This work was supported by the Natural Sciences and Engineering Research Council of Canada. One of us (G.W.F.D.) is grateful to the Killam Foundation for support.
[1] H. Kleindienst and S. Beutner, Chem. Phys. Lett. 164, 291 (1989).

[2] F. W. King, Phys. Rev. A 40, 1735 (1989), and earlier references therein.

[3] F. W. King and M. P. Bergsbaken, J. Chem. Phys. 93, 2470 (1990).

[4] K. T. Chung, Phys. Rev. A 44, 5421 (1991).

[5] O. Jitrik and C. F. Bunge, Phys. Rev. A 43, 5804 (1991).

[6] G. W. F. Drake, Phys. Rev. Lett. 65, 2769 (1990); Nucl. Instrum. Methods Phys. Res. Sect. B 31, 7 (1988).

[7] S. Larsson, Phys. Rev. 169, 49 (1968).

[8] C. F. Bunge, Phys. Rev. A 16, 2496 (1977).

[9] C. E. Moore, Ionization Potentials and Ionization Limits Derived from the Analysis of Optical Spectra, Natl. Bur. Stand. (U.S.) Ref. Data Ser. No. 34 (U.S. GPO, Washington, DC, 1970).

[10] D. E. Freund, B. D. Huxtable, and J. D. Morgan III, Phys. Rev. A 29, 980 (1984).

[11] G. W. F. Drake, Can. J. Phys. 66, 586 (1988).

[12] S. P. Goldman and G. W. F. Drake, J. Phys. B 17, L197 (1984).
[13] P. Indelicato and J. P. Desclaux, Phys. Rev. A 42, 5139 (1990), and earlier references therein.

[14] W. R. Johnson and G. Soff, At. Data Nucl. Data Tables 33, 405 (1985).

[15] A. Dalgarno and E. M. Parkinson, Phys. Rev. 176, 73 (1968).

[16] C. L. Pekeris, Phys. Rev. 112, 1649(1958).

[17] C. J. Sansonetti, J. D. Gillaspy, and C. L. Cromer, Phys. Rev. Lett. 65, 2539 (1990).

[18] W. Lichten, D. Shiner, and Z.-X. Zhou, Phys. Rev. A 43, 1663 (1991).

[19] K. T. Cheng, W. R. Johnson, and J. Sapirstein, Phys. Rev. Lett. 66, 2960 (1991).

[20] The experimental value is derived in Ref. [18] from the measurement by J. Schweppe, A. Belkacem, L. Blumenfield, N. Claytor, B. Feinberg, H. Gould, V. E. Kostroun, L. Levy, S. Misawa, J. R. Mowat, and M. H. Prior, Phys. Rev. Lett. 66, 1434 (1991)

[21] W. R. Johnson, S. A. Blundell, and J. Sapirstein, Phys. Rev. A 37, 2764 (1988). 